\title{
Mandibular Second Molar Protraction with Temporary Anchorage Devices-A Case Report
}

\author{
Anilkumar Katta ${ }^{1, *}$, K. Karthik ${ }^{2}$, Venkataramana Vannala ${ }^{3}$, N. Kurinji Kumaran ${ }^{2}$, Revathi Peddu ${ }^{1}$, \\ Vaishnavi Dasari ${ }^{4}$ \\ ${ }^{1}$ Department of Orthodontics and dentofacial orthopaedics, Sibar Institute of Dental Sciences, Takkellapadu, Guntur, Andhra Pradesh \\ ${ }^{2}$ Department of Orthodontics and dentofacialorthopaedics, Raja Muthiah Dental College and Hospital, Annamalai University, \\ Chidambaram, Tamilnadu \\ ${ }^{3}$ Department of Orthodontics, PanineeyaMahavidhyalaya Institute of Dental Sciences, Kamalanagar, Hyderabad, Telangana, A.P. India \\ ${ }^{4}$ Department of Conservative dentistry \&Endodontics, Saint Joseph dental college, Eluru, Andhra Pradesh, India \\ *Corresponding author: anilchow82@gmail.com
}

Received October 05, 2014; Revised October 25, 2014; Accepted October 28, 2014

\begin{abstract}
Edentulous spaces can be closed with dental implants, prosthesis, and orthodontic space closure. As mandibular bone is dense, protraction of molars is time taking and challenging. Temporary anchorage devices play a vital role in providing anchorage. Translation of molars can be achieved with power arms which are placed near to the centre of resistance. This is a case with bilaterally missing mandibular first molars and lower central incisor. Ridge defect is seen in molar region on right side. Class II molar relation was achieved bilaterally with second molar moving into first molar space followed by the third molar. Translation was achieved bilaterally and $10 \mathrm{~mm}$ of space closure was done with the help of TAD's and power arms.
\end{abstract}

Keywords: translation, $T A D$, power arm

Cite This Article: Anilkumar Katta, K. Karthik, Venkataramana Vannala, N. Kurinji Kumaran, Revathi Peddu, and Vaishnavi Dasari, "Mandibular Second Molar Protraction with Temporary Anchorage Devices-A Case Report.” International Journal of Dental Sciences and Research, vol. 2, no. 6A (2014): 1-4. doi: 10.12691/ijdsr-2-6A-1.

\section{Introduction}

The most commonly missing teeth in the oral cavity are mandibular first molars due to caries [1]. In a case with missing molars where orthodontic treatment is necessary protraction of second molars into first molar space will be a good option with proper bone support and oral hygiene. Molar protraction generally results in anchorage loss, tipping of the molars and root resorption [2]. Molar protraction with the help of TAD is effective and prevents anchorage loss which is frequently used in critical anchorage cases [3]. Simultaneous anterior retraction and posterior protraction is achieved, but to move a molar in the mandibular arch is difficult, due to its bone density which result in much of anterior retraction than of posterior protraction. Translation of molars can be achieved with the help of power arms which are placed near to the centre of resistance of the molars ${ }^{4}$. Mandibular molars tend to tip, as the force acts away from the centre of resistance. Placing a power arm in the accessory buccal tube helps in acting the force near to the centre of resistance which helps in translation.

\section{Diagnosis and Treatment Objectives}

Young women aged 20 years came to the department with a chief complaint of proclined upper anteriors and missing posterior teeth in the lower arch.

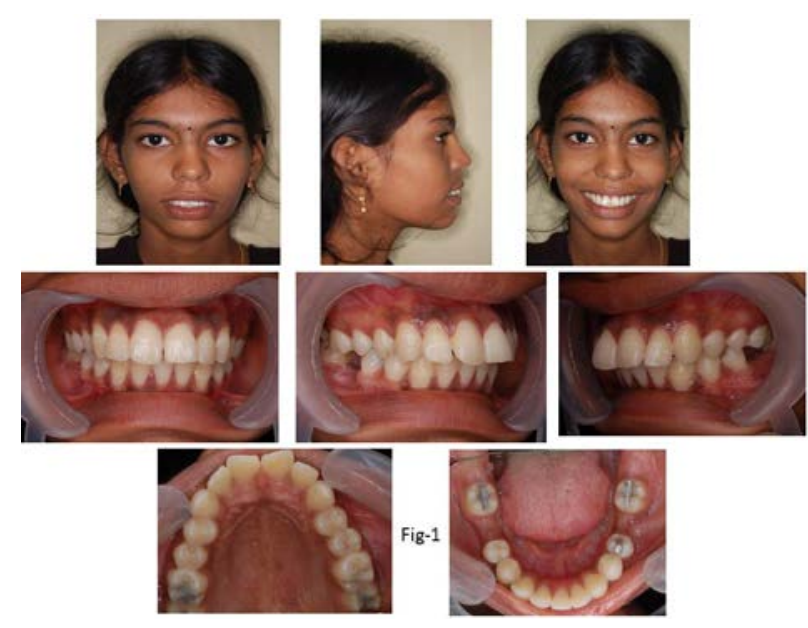

Figure 1.

On clinical and radiographic examination the patient presented class I canine relation with orthognathic maxilla and mandible (Figure 1, Figure 2). Upper anteriors are proclined with an overjet of $6 \mathrm{~mm}$ and overbite of $3.5 \mathrm{~mm}$. Lower right central incisor and mandibular first molars are missing bilaterally. Panoramic radiograph revealed erupting third molars on both the sides and a class III 
ridge defect was seen in the missing molar region on the right side (Figure 3).

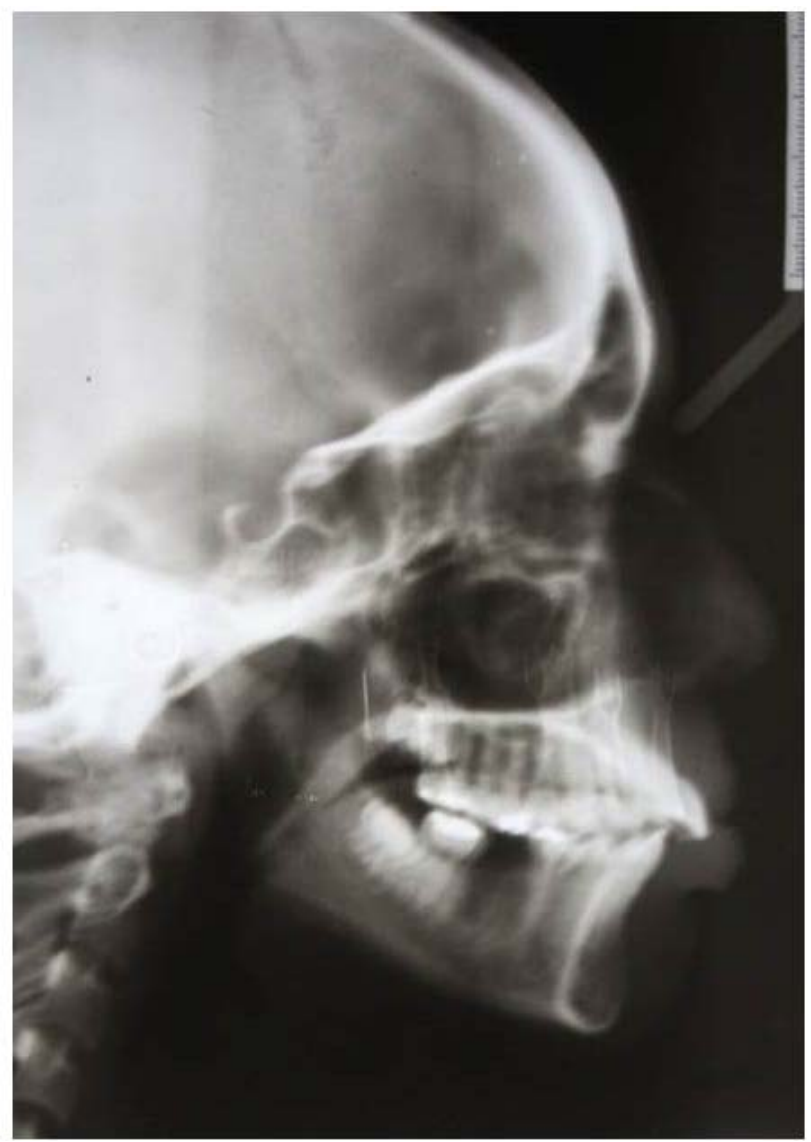

Figure 2.

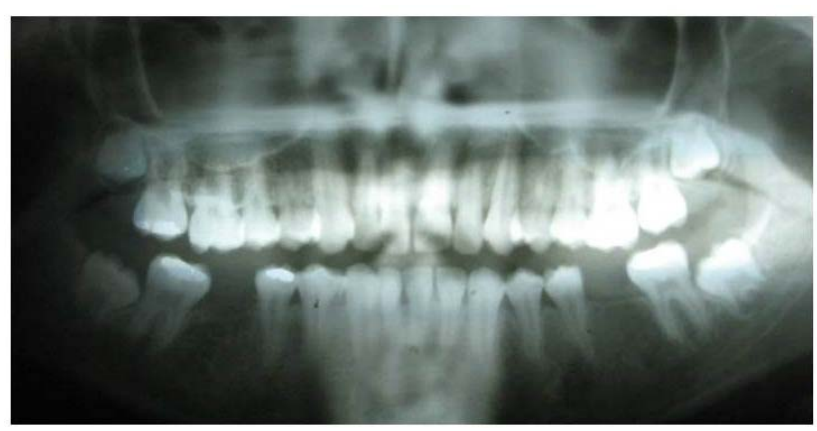

Figure 3.

When explained about different modes of treatment like endosseous implants, prosthesis and orthodontic appliance to close the posterior spaces, patient has opted to close the spaces by orthodontic tooth movement.

Treatment objectives were to correct the proclination of upper anteriors by extracting first premolars, and planned to maintain the maxillary molars in the same position as this is a case of critical anchorage mini implants are used to retract the upper anteriors by retaining the molars in the same position. In the lower arch as patient was not willing for prosthetic bridges or to undergo surgery for the placement of endosseous implant, it was planned to protract the molars bilaterally with the help of TAD's. The final outcome was to achieve Class II molar relation and maintain class I canine relation.
Preadjusted edge wise appliances with 0.022 slots were bonded to upper and lower arch, levelling and aligning was accomplished with 0.016 niti wire in upper and lower arch (Figure 4). The sequence of wires used was 0.016x0.022 niti, 0.017x0.025 niti, and 0.019x0.025 niti. After finishing with niti wires, space closure was done on $0.019 \times 0.025$ stainless steel wire in upper and lower arch. Temporary anchorage devices $(6 \mathrm{~mm}$ long and $1.5 \mathrm{~mm}$ diameter) are placed distal to the first molar in the upper arch on the buccal side and distal to the canine in the lower arch by self-drilling method under local anesthesia (Figure 5). Power arms are fabricated with 0.017x0.025 stainless steel wire, placed into the auxillary slot of the buccal tube and cinched tightly. Two weeks after the placement of orthodontic mini screws, closed coil springs of $9 \mathrm{~mm}$ length are used to protract the molars in the lower arch and retract the anteriors in the upper arch (Figure 6).

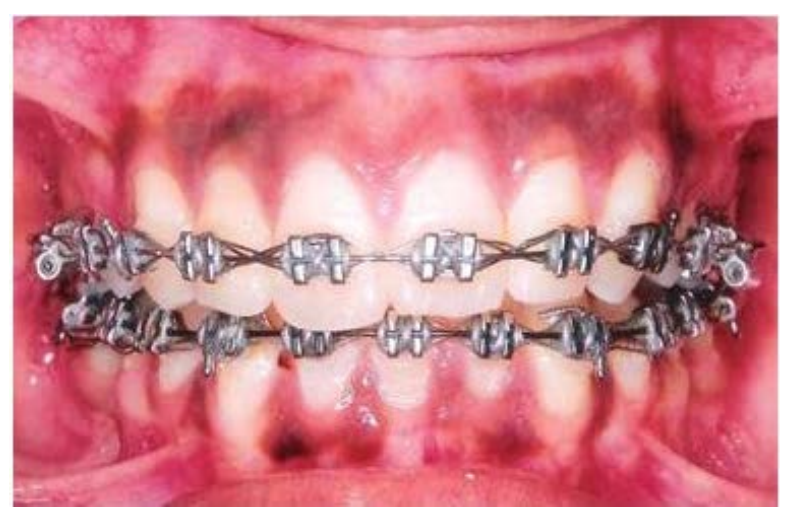

Figure 4.

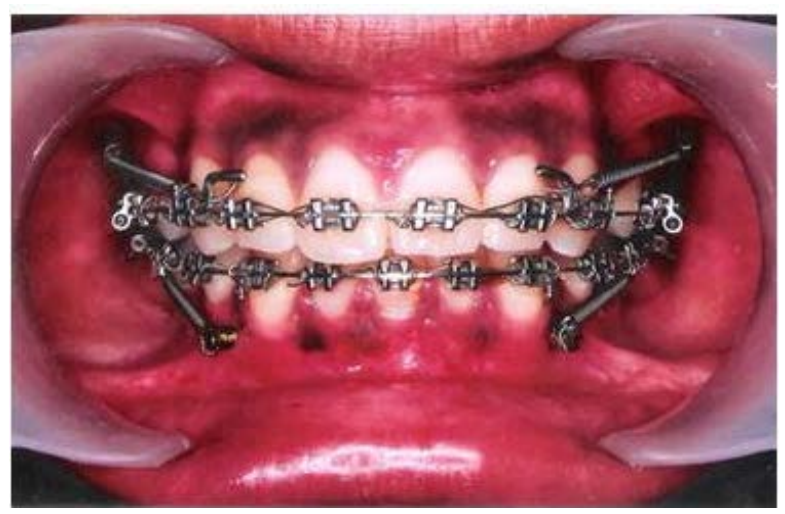

Figure 5.

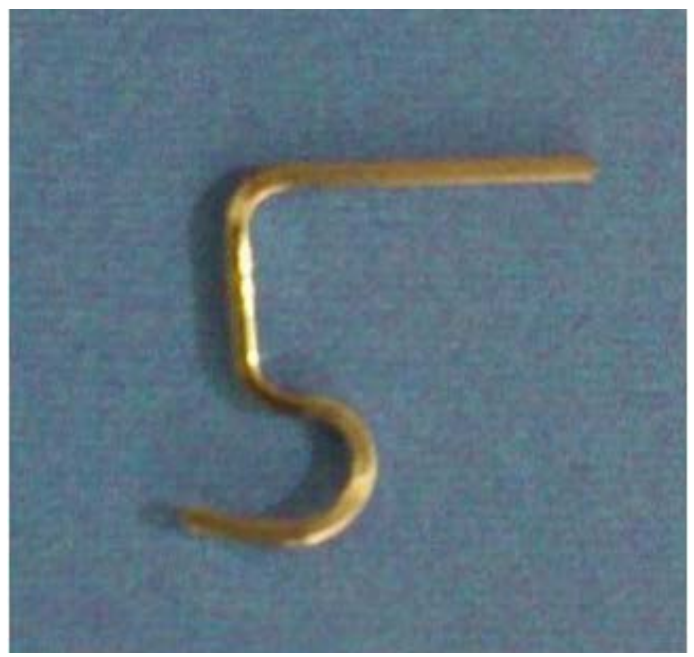

\section{Treatment Progress}

Figure 6. 


\section{Treatment Results}

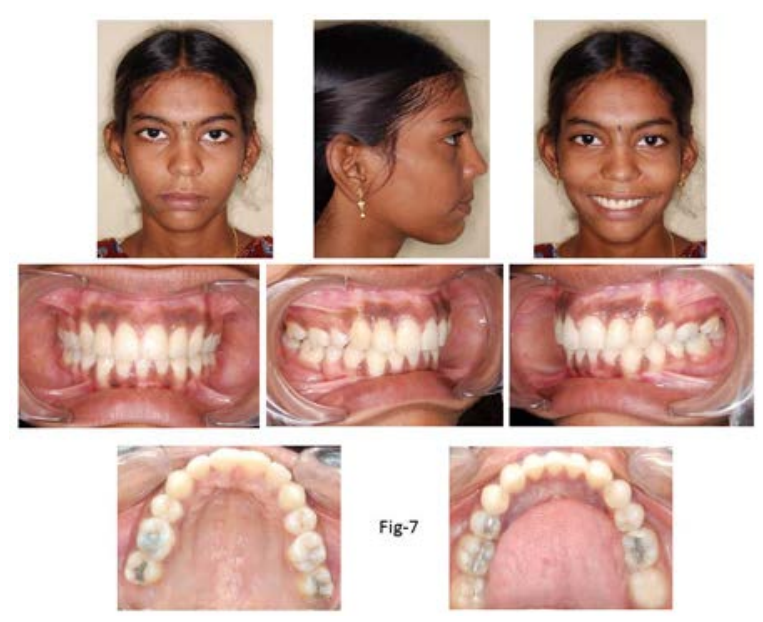

Figure 7.

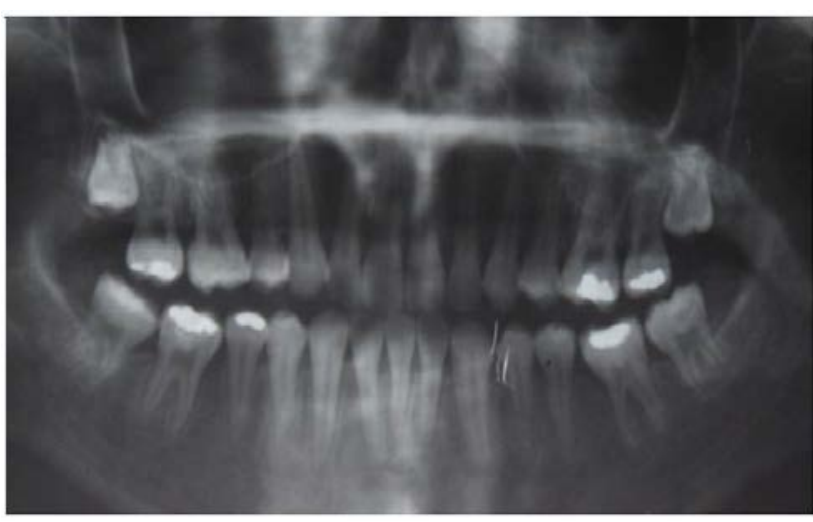

Figure 8.

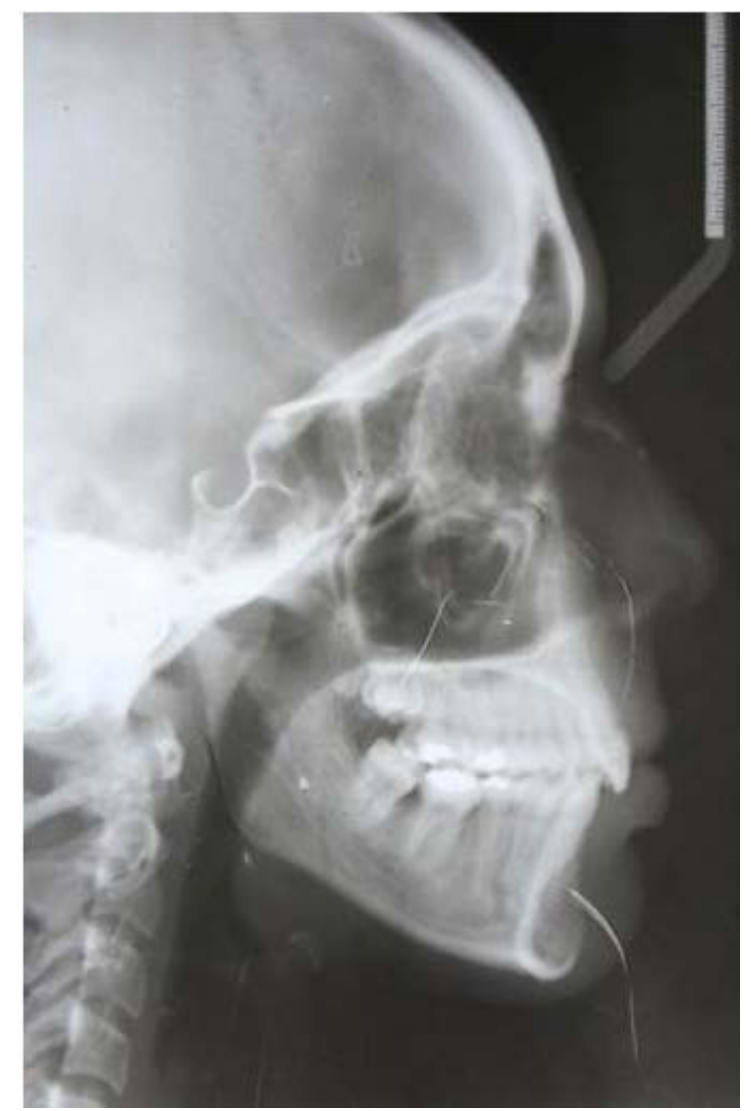

Figure 9.
The planned treatment objectives were achieved with well aligned upper and lower teeth. Overjet and overbite came to normal, class II molar relation was achieved on both the sides and class I canine relation was retained. The edentulous spaces in the lower arch were completely closed by protraction of molars in the lower arch, $10 \mathrm{~mm}$ on the right side and $9.5 \mathrm{~mm}$ on the left side (Figure 7). Post treatment panoramic radiograph showed good amount of root parallelism and decent amount of alveolar bone height, in spite of ridge defect (Figure 8). Post treatment cephalogram showed mesial movement of second and third molars (Figure 9). Fixed lingual retainers were bonded on to the upper and lower arch.

\section{Discussion}

Protraction of molars in the lower arch is time consuming and challenging due to thick density of mandibular bone. Anchorage in orthodontics is crucial; many auxillary appliances are developed in the process of conserving anchorage with their own limitations. With the advent of TAD's or mini implants anchorage was not a concern in achieving any kind of tooth movement. Today in orthodontics all kinds of tooth movements like protraction, retraction, expansion, intrusion and extrusion are achieved easily with the help of mini implants [5]. Accurate care should be taken while placing the implants, starting from site selection. The amount of failure rate was also high in the mandible due to close proximity of roots and heavy musculature of mandible, post extraction alveolar ridge resorption was higher in the mandible than maxilla [6].

TAD's are placed bucally in between first and second molar in the upper arch and distal to the canine in the lower arch by self drilling method. Closed coil springs are used to retract in the upper arch and lower arch so that it provides constant and continuous force, as there won't be any force decay [7]. In the upper arch they are activated with the ligature wire and tied into the L hook anteriorly, which was fabricated with $0.019 \times 0.025$ stainless steel wire and into the mini implant posteriorly. In the lower arch closed coil springs are tied to the mini implant anteriorly and to the power arm posteriorly.

Power arms are fabricated with $0.017 \times 0.025$ stainless steel wire. The length of the power arm varies individually, and care should be taken to not to impinge into the buccal sulcus of the patient. Power arms are placed in the accessory buccal tube of second molars bilaterally in the lower arch, from the distal aspect of the second molar. The hook should face posteriorly, as there won't be any escape of the ligature wire from the hook and cinched tightly on the anterior end of the buccal tube. The centre of reistance of molars is located at the furcation area. Power arms placed cervically, near to the furcation area [8]. Forces acting at the centre of resistance produce bodily movement. Molars tend to tip easily when forces are applied at the buccal tube which is away from the centre of reistance.

Siebert classification was used to diagnose edentulous ridge resorption [9] (Figure 10). The present case has Siebert class III ridge defect which is a combination of both buccolingual and apicoronal loss of hard and soft tissue, which was seen clinically and on panoramic 
radiograph. Protraction of molars was done as there was sufficient bone to support the molar and the concept of ridge augmentation where deposition and resorption occurs during orthodontic tooth movement.
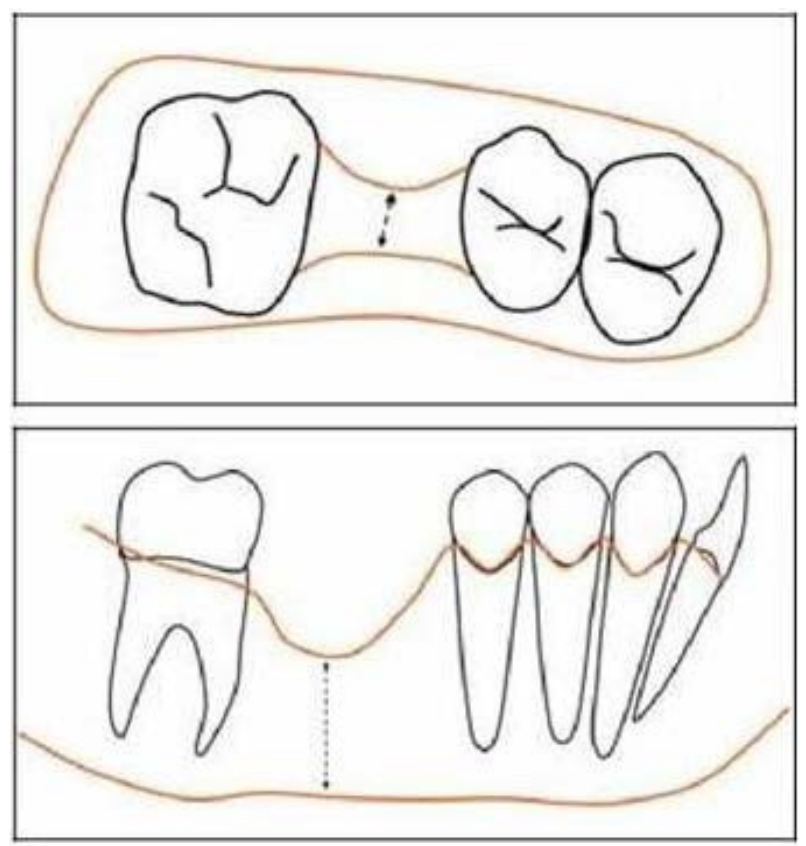

Figure 10.

Mandibular ridge gets wider while going posteriorly. Protraction of molars leads to cross bite, because from the wider area we are moving a molar into the narrower area. The arch wire was expanded posteriorly in every visit to compensate for the cross bite effect.

The duration of the treatment was 36 months, $10 \mathrm{~mm}$ of molar protraction was done on the right side and $9.5 \mathrm{~mm}$ on the left side. Mid line shift was seen in the frontal photograph due to the missing incisor in the lower arch. Root paralleling was decent and root resorption was insignificant, as seen on the post treatment panoramic radiograph and lateral cephalogram. Upper molars were intruded slightly as they were supra erupted due to long duration of edentulous ridge. Fixed lingual retainer was given in the upper and lower arch, Hawley retainer was also used in both the arches.

Conclusion: Protraction of molars with TAD's in case of missing first molars is a cost effective treatment. Mini implants are easy to place and can achieve all kinds of tooth movement. Power arms produce translation, when they are placed for anterior retraction or posterior protraction. Protraction of second molars into the first molar space is a time taking procedure and should be evaluated on case to case individually.

\section{References}

[1] Roberts WE, Hall WB, Gluskin AH. Edentulous spaces in the mandibular posterior segments. In: Mosby 1998: 177-179.

[2] Stepovich M1 A clinical study on closing edentulous spaces in the mandible. Angle orthod 1979; 49; 227-233.

[3] Kim SH, Kook YA, Jeong DM etal. Clinical application of accelerated osteogenic orthodontics and partially osseointegrated mini implants for minor tooth movement. Am J Orthod Dentofacial Orthop. 2009. 136; 431-439.

[4] Ansari TA, Mascarenhas R, Hussain A etal. Evaluation of the power arm in bringing about bodily movement using finite element analysis. Orthodontics (chic). 2011 winter; 12(4): 318-29.

[5] Sung JH, Kyung HM, Bae SM et al. Microimplants in orthodontics. Dentos, Daegu, Korea. 2006; 70.

[6] Neal D. Kravitz, Tyler Jolley. Mandibular molar protraction with temporary anchorage devices. JCO 2008. Vol XLII number 6.

[7] C. Nightingale, S.P.Jones. A clinical investigation of force delivery systems for orthodontic space closure. Journal of orthodontics. September 2003, vol 30, no 3. 229-236.

[8] Ioannis S. Papageorgiou. The centre of resistance of teeth in orthodontics. Hell Orthod review 2005; 8: 41-55.

[9] Seibert, J.S. Reconstruction of deformed, partially edentulous ridges, using full thickness onlay grafts, part I: Technique and wound healing, compend. Cont.Ed. Dent.4: 437-453, 1983. 